Research Article

\title{
On the Resolution of a Remarkable Bond Pricing Model from Financial Mathematics: Application of the Deductive Group Theoretical Technique
}

\author{
Taha Aziz \\ Department of Mathematics, Dammam Community College, King Fahd University of Petroleum and Minerals, \\ KFUPM Box 5084, Dhahran 31261, Saudi Arabia
}

Correspondence should be addressed to Taha Aziz; tahaaziz77@yahoo.com

Received 16 March 2021; Accepted 7 June 2021; Published 15 June 2021

Academic Editor: Kamal Shah

Copyright (c) 2021 Taha Aziz. This is an open access article distributed under the Creative Commons Attribution License, which permits unrestricted use, distribution, and reproduction in any medium, provided the original work is properly cited.

\begin{abstract}
The classical Cox-Ingersoll-Ross (CIR) bond-pricing model is based on the evolution space-time dependent partial differential equation (PDE) which represents the standard European interest rate derivatives. In general, such class of evolution partial differential equations (PDEs) has generally been resolved by classical methods of PDEs and by ansatz-based techniques which have been previously applied in a similar context. The author here shows the application of an invariant approach, a systematic method based on deductive group-theoretical analysis. The invariant technique reduces the scalar linear space-time dependent parabolic PDE to one of the four classical Lie canonical forms. This method leads us to exactly solve the scalar linear space-time dependent parabolic PDE representing the CIR model. It was found that CIR PDE is transformed into the first canonical form, which is the heat equation. Under the proper choice of emerging parameters of the model, the CIR equation is also reduced to the second Lie canonical form. The equivalence transformations which map the CIR PDE into the different canonical forms are deduced. With the use of these equivalence transformations, the invariant solutions of the underlying model are found by using some well-known results of the heat equation and the second Lie canonical form. Furthermore, the Cauchy initial-value model of the CIR problem along with the terminal condition is discussed and closed-form solutions are deduced. Finally, the conservation laws associated with the CIR equation are derived by using the general conservation theorem.
\end{abstract}

\section{Theory and Background}

The bond-pricing theory began in the 19th century when Bachelier [1] gave the assumption that the pricing of bond depends on stock price which follows a Brownian motion. The pioneering work of Merton [2] and later by Black and Scholes [3] also gave a new dimension to mathematical formulation of finance problems. The actual mathematical formulation of these models was initially given in the form of stochastic differential equations (DEs), but after incorporating certain assumptions, these models were derived in terms of parabolic PDEs with variable coefficients. The fundamental model of bond-pricing theory was first proposed by Vasicek [4], which was modified by Cox et al. in [5], now famous in literature as the CIR bond-pricing model.
For the Vasicek model, the instantaneous rate $z(t)$ is represented by an Ornstein-Uhlenbeck process, which is written in the form $[4,5]$ :

$$
\mathrm{dz}(t)=\beta \mathrm{dW}(t)+\mu(\alpha-z(t)) \mathrm{dt},
$$

with $W(t)$ is the standard Brownian motion and $\mu(\alpha-z(t))$ is the drift term. For the CIR model, the instantaneous rate followed a stochastic process of the form

$$
\mathrm{dz}(t)=\beta \sqrt{z(t)} \mathrm{dW}(t)+\mu(\alpha-z(t)) \mathrm{dt} .
$$

The mean-reversion feature of these bond-pricing models is consistent with economic phenomenon. However, the main disadvantage of the Vasicek model is that it allows the negative interest rates. On the contrary, the CIR model provides improvement in the Vasicek model by ensuring 
that the interest rate can never be negative. The CIR model, therefore, incorporates the number of important characteristics of interest rate models.

For Vasicek model, the price bond $F(z, t)$ satisfies the following parabolic equation $[6,7]$ :

$$
F_{t}+\frac{1}{2} \beta^{2} F_{z z}+\mu(\alpha-z) F_{z}-z F=0
$$

In the CIR model, the price of a zero-coupon $F(z, t)$ satisfies the parabolic $(1+1) \operatorname{PDE}[6,7]$, which is written as

$$
F_{t}+\frac{1}{2} \beta^{2} z F_{z z}+\mu(\alpha-z) F_{z}-z F=0 .
$$

For both the above PDEs, the value $F(z, t)$ of the zerocoupon bond matures at time $t=T$ with a value of unity, i.e.,

$$
F(z, T)=1
$$

which is known as terminal condition, where $T$ denotes the expiration time and gives the payoff of the derivative on maturity.

A mapping has been constructed in [8] which embeds the Vasicek model into the CIR model such that, under this mapping, any solution of the CIR equation can be transformed into a solution of the Vasicek equation. The solutions of the CIR equation are, therefore, rather more general and provide deeper insights into interest rate models.

It is important to note that the general PDE in which both Vasicek equation (3) and CIR equation (4) are embedded is given by

$$
F_{t}+\frac{1}{2} \beta^{2} F_{\mathrm{zz}}+(F-\alpha \beta) F_{z}-z F=0
$$

The above equation models the price of a zero-coupon bond when the spot rate is determined by the general stochastic process:

$$
\mathrm{dz}(t)=\beta(x, t) \mathrm{dW}(t)+F(z, t) \mathrm{dt} .
$$

The function $\alpha(z, t)$ in equation (7) represents the market price of risk. The particular cases of PDE (10) have been considered in [9], in which the authors performed the group classification of the general equation by using some simplifying assumptions. The group invariant solutions of the CIR equation (4) were first found in $[10,11]$. The complete Lie symmetry group admitted by (4) was found for all choices of the parameters. It was found that, besides trivial symmetries, some additional symmetries were obtained. The self-similar solutions of PDE (4) subject to the terminal condition (5) were deduced for special parametric choices (refer to $[10,11]$ ).

The applications of Lie groups to problems in mathematical finance are not new, and it is certainly young enough, with a possibility to expand. Generally, the nonlinear DEs that arise in mathematical finance are usually tackled with either approximate or numerical methods. Whereas, the Lie symmetry approach allows us to find complete local one-parameter transformation groups that can be used to find exact solutions or to reduce nonlinear DEs to linear DEs. Concerning the relationship between Lie symmetry methods and financial mathematics and economic models, we would like to note the presence of several recent studies [12-22], which represent the applications and utility of well-known concepts of Lie theory to several problems from mathematical finance.

Lie [23] was the first to introduce the classification of DEs in terms of continuous groups. Lie classified the scalar second-order $(1+1)$ PDEs and developed a method for their integration. Lie provided all the canonical forms of the scalar linear $(1+1)$ PDE for which the equation admits six-, four-, two-, and one-dimensional nontrivial symmetry algebras. Ovsiannikov [24] further investigated the parabolic linear $(1+1)$ PDE by using the reduction to the fourth Lie canonical form. Bluman in [25] studied the symmetry properties of the parabolic linear $(1+1)$ PDE and developed a mapping algorithm. Johnpillai and Mahomed [26] were the first to propose the invariant approach to Fokker-Planck equations. They proved that the linear $(1+1)$ parabolic equation was reducible to the heat equation via a local equivalence transformation. The necessary and sufficient conditions were discussed in [26], for a scalar linear $(1+1)$ parabolic equation which can be reduced to a one-dimensional heat equation through equivalence transformations. A few years later, Mahomed [27] discussed the complete invariant characterization of scalar linear $(1+1)$ parabolic PDE into four canonical forms. The refined invariant criteria, for the reduction of $(1+1)$ parabolic equation into different canonical forms, in terms of the coefficients of the parabolic equation were derived in [27]. Recently, the usefulness of the invariant criterion has been thoroughly demonstrated in studies [28-30].

From the definition point of view, a general scalar $(1+1)$ PDE is written as

$$
F_{t}=A(t, z) F_{\mathrm{zz}}+B(t, z) F_{z}+C(t, z) F,
$$

with $A(t, z), B(t, z)$, and $C(t, z)$ are continuously differentiable functions. The equivalence transformations of this PDE are an infinite local group of linear transformations of the dependent variables, given as

$$
\bar{F}=D(t, z) F .
$$

Together with equivalence transformations of independent variables,

$$
\bar{t}=f(t), \bar{z}=W(t, z), f_{t} \neq 0, W_{x} \neq 0,
$$

where $f(t), D(t, z)$, and $W(t, z)$ are arbitrary functions. Two parabolic equations of the form given in equation (8) are considered to be equivalent only if they can be mapped to one another by the suitable combinations of equivalence transformations. Lie was the first to deduce that every linear scalar $(1+1)$ parabolic PDE can be mapped on the following four canonical forms: 


$$
\begin{aligned}
& F_{t}=F_{\mathrm{zz}}, \\
& F_{t}=F_{\mathrm{zz}}+\frac{A}{z^{2}} F, \quad a \neq 0, \\
& F_{t}=F_{\mathrm{zz}}+C(z) F, \quad c \neq 0, \\
& F_{t}=F_{\mathrm{zz}}+C(t, z) F, \quad c \neq 0 .
\end{aligned}
$$

The first form is the classical heat PDE which contains six nontrivial symmetry generators. The second canonical equation has four nontrivial symmetry generators, the third generally has two symmetry generators, and the fourth has one nontrivial symmetry generator. The following theorems give the algorithm on the invariant characterization of scalar $(1+1)$ parabolic PDE into different canonical forms given in equation (11).

Theorem 1. The scalar $(1+1)$ parabolic PDE (8) can be reduced to the heat equation by a transformation if and only if the coefficients in equation (8) satisfy the condition

$$
2 l_{z}+2 m_{z}-n_{z}=0
$$

where

$$
\begin{aligned}
& l=|A|^{1 / 2}\left[|A|^{1 / 2} \Pi_{z}\right]_{z}, m=|A|^{1 / 2}\left[|A|^{1 / 2} \partial_{t}(B / 2 A)\right]_{z} \\
& n=|A|^{1 / 2} \partial_{t}^{2}\left(1 /|A|^{1 / 2}\right)
\end{aligned}
$$

with $\Pi$ given by

$$
\Pi=C-\frac{B_{z}}{2}+\frac{B A_{z}}{2 A}+\frac{A_{z z}}{4}-\frac{3}{16} \frac{A_{z}^{2}}{a}-\frac{A_{t}}{2 A}-\frac{B^{2}}{4 A} .
$$

Theorem 2. The scalar linear $(1+1)$ parabolic PDE (8) is reducible to the second Lie canonical equation if and only if the coefficients in equation (8) satisfy the following invariant equation:

$$
\begin{aligned}
20 l_{z} & +20 m_{z}-10 n_{z}+10\left[|A|^{1 / 2} m_{z}\right]_{z} \\
& \int \frac{d z}{|A|^{1 / 2}}-5\left[|A|^{1 / 2} n_{z}\right]_{z} \int \frac{d z}{|A|^{1 / 2}} \\
& +10\left[|A|^{1 / 2} l_{z}\right]_{z} \int \frac{d z}{|A|^{1 / 2}}+\left[|A|^{1 / 2}\left[|A|^{1 / 2} l_{z}\right]_{z}\right]_{z}\left(\int \frac{d z}{|A|^{1 / 2}}\right)^{2} \\
& +\left[|A|^{1 / 2}\left[|A|^{1 / 2} m_{z}\right]_{z}\right]_{z}\left(\int \frac{d z}{|A|^{1 / 2}}\right)^{2} \\
& -\frac{1}{2}\left[|A|^{1 / 2}\left[|A|^{1 / 2} n_{z}\right]_{z}\right]_{z}\left(\int \frac{d z}{|A|^{1 / 2}}\right)^{2}=0
\end{aligned}
$$

with $l, m, n$, and $\Pi$ are defined in equations (13) and (14).

Theorem 3. The parabolic PDE (8) reduces to the classical heat PDE by the transformation relations:

$$
\begin{aligned}
\bar{t}= & \chi(t), \\
\bar{z}= & \pm \int\left[\dot{\chi} A(t, z)^{-1}\right]^{1 / 2} d z+\sigma(t), \\
\bar{F}= & f(t)[A(t, z)]^{-1 / 4} F \exp \left[\int \frac{B(t, z)}{2 A(t, z)} d z-\frac{1}{8} \frac{\ddot{\chi}}{\dot{\chi}}\left(\int \frac{d z}{A(t, z)^{1 / 2}}\right)^{2}\right. \\
& \left.-\frac{1}{2} \int \frac{1}{A(t, z)^{1 / 2}} \frac{\partial}{\partial t}\left(\int \frac{d z}{A(t, z)^{1 / 2}}\right) d z \pm \frac{1}{2} \frac{\dot{\sigma}}{\dot{\sigma}^{1 / 2}} \int \frac{d z}{A(t, z)^{1 / 2}}\right],
\end{aligned}
$$

where $\chi, \sigma$, and $f$ satisfy the relation

$$
\begin{aligned}
\dot{\chi} \bar{c}= & \Pi+\frac{\partial}{\partial t} \int \frac{B(t, z)}{2 A(t, z)} d z-\frac{1}{2} \int \frac{1}{A(t, z)^{1 / 2}} \frac{\partial^{2}}{\partial t^{2}}\left(\int \frac{d z}{A(t, z)^{1 / 2}}\right) d z \\
& +u(t)\left(\int \frac{d z}{A(t, z)^{1 / 2}}\right)^{2}+v(t)\left(\int \frac{d z}{A(t, z)^{1 / 2}}\right)+w(t),
\end{aligned}
$$

where $\Pi$ is defined in equation (14) and

$$
\begin{aligned}
& u(t)=\frac{1}{16} \frac{\ddot{\chi}^{2}}{\dot{\chi}^{2}}-\frac{1}{8}\left(\frac{\ddot{\chi}}{\dot{\chi}}\right)_{t}, \\
& v(t)= \pm \frac{1}{4} \frac{\ddot{\chi}}{\dot{\chi}} \frac{\dot{\sigma}}{\dot{\chi}^{1 / 2}} \pm \frac{1}{2}\left(\frac{\dot{\sigma}}{\dot{\chi}^{1 / 2}}\right)_{t}, \\
& w(t)=\frac{1}{4} \frac{\ddot{\chi}}{\dot{\chi}}+\frac{1}{4} \frac{\dot{\sigma}^{2}}{\dot{\chi}}+\frac{\dot{f}}{f} .
\end{aligned}
$$

The functions $u, v$, and $w$ are constrained by relation (18).

The derivation of conservation laws plays an important role in understanding the mathematical models described by DEs. For several mathematical and physical models, the conservation laws are used to study the existence and uniqueness of solutions of nonlinear DEs. The conservation laws are applied in the case of PDEs to search for possible potential symmetries and related nonclassical symmetries. Consequently, it is necessary to derive the conservation laws of the CIR equation, arguably one of the most popular mathematical finance models. There are many approaches available to the construction of conservation laws for PDEs [31-37]. The classical Noether theorem provides a sophisticated way to construct conservation laws once the Lagrangian associated with Noether symmetries are found for the corresponding Euler-Lagrange equation. Recently, a general result has been established on the conservation laws for arbitrary DEs, which is based on the concept of an adjoint equation for associated DE [38]. According to this result, it is possible to find a conservation law with any group of Lie symmetries and to derive conservation laws without seeking classical Lagrangians.

Motivated by the facts stated above, the purpose of this paper is to describe the systematic application of invariant criterion to precisely solve the traditional CIR model from mathematical finance. The majority of financial interest rate problems are modeled using linear parabolic $(1+1)$ PDEs. 
Because many of the DEs in the literature addressing financial models are highly rich in symmetry, the Lie group theory of transformation groups is widely useful. As a result, the remainder of the study demonstrates the algebraic features of the CIR model in the context of an invariant criterion based on Lie groups. The closed-form solutions will be derived for the model problem under a suitable choice of terminals condition. By not using the ansatz method or relying on adhoc approach, this will be achieved through an algorithmic technique: the invariant criterion.

\section{Fundamental Solutions of Cox-Ingersoll-Ross Bond-Pricing Equation}

The fundamental solutions of CIR bond-pricing equation under the implication of invariant criterion are constructed in this section. Two separate cases will be considered for the reduction of CIR equation (4) into heat equation and second canonical equation. Thereafter, the invariant solutions of the model are deduced.

2.1. Case 1: Reduction to Heat Equation $-F_{t}=F_{z z}$. First, find the equivalence transformations which reduce equation (4) into different canonical forms. With the comparison of PDE (4) with scalar linear parabolic PDE (8), the coefficients functions are given by

$$
\begin{aligned}
& A(t, z)=-\frac{1}{2} \beta^{2} z, \\
& B(t, z)=-\mu(\alpha-z), \\
& C(t, z)=z .
\end{aligned}
$$

With the use of Theorem 1, the parametric values are deduced such that the PDE (4) is mapped to the heat equation. The value of $\Pi$ given in equation (14) for the CIR equation is found as

$$
\Pi=\frac{\mu^{2}(z-\alpha)^{2}}{2 \beta^{2} z}+\frac{\mu(z-\alpha)}{2 z}+\frac{3}{32} \frac{\beta^{2}}{z}-\frac{\mu}{2}+z .
$$

It is clear that $m$ and $n$ are equal to zero in the condition defined in Theorem 1 because the coefficients of PDE (4) are independent of time $t$. Under the value of $\Pi$, condition (12) takes the form

$$
l_{z}=z^{3}\left[-\frac{3}{8} \beta^{4}+2 \mu \alpha \beta^{2}-2 \mu^{2} \alpha^{2}\right] .
$$

From the above equation, one obtains the following parametric conditions:

$$
\beta_{1}=-\sqrt{\frac{4 \mu \alpha}{3}}, \beta_{2}=\sqrt{\frac{4 \mu \alpha}{3}}, \beta_{3}=-\sqrt{4 \mu \alpha}, \beta_{4}=\sqrt{4 \mu \alpha} .
$$

For specific values of the parameter $\beta$, equation (22) gives the nontrivial cases such that invariant condition (12) is holds, which reduces the CIR PDE (4) to the heat equation.

Now, the transformation relations will be computed which give reduction of the CIR equation (4) to the heat equation. These transformations are further used to find the fundamental solutions of the PDE (4).

With the use of the coefficients as defined in Theorem 3, one obtains the following results:

$$
\begin{aligned}
& u(t)=-\frac{1}{8}\left(\beta^{2}+\frac{\mu^{2}}{2}\right), v(t)=0, w(t)=\frac{\mu^{2} \alpha}{\beta^{2}}, \chi(t)=k_{1} \sqrt{\frac{2}{K}} \tan \left(\sqrt{\frac{K}{2}} t\right), \\
& \sigma(t)=k_{2} \int \frac{d t}{\ln \cos (\sqrt{K / 2} t)}, \\
& f(t)=\exp \left[\frac{1}{2} \ln \cos (\sqrt{K / 2} t)-\frac{k_{3}^{2}}{4} \int \frac{d t}{[\ln \cos (\sqrt{K / 2} t)]^{2}}+t+k_{4}\right],
\end{aligned}
$$

where $K=8 f(t)$ and $k_{j}(j=1,2,3,4)$ are the integration constants and $\beta$ can take any value as given in equation (22). Substituting the values from equation (23) to equation (16), the equivalence transformations which give the reduction of CIR equation (4) into the heat equation (after performing some lengthy and tedious calculation) are given as

$$
\begin{aligned}
\bar{t}= & k_{1} \sqrt{2 / K} \tan (\sqrt{2 / K} t), \\
\bar{z}= & \pm \frac{\sqrt{4 k_{1}}}{\cos (\sqrt{2 / K} t)} \sqrt{z}+k_{2} \int_{t}^{T} \frac{d t}{\ln \cos (\sqrt{2 / K} t)}+k_{3}, \\
\bar{F}= & \left(\frac{1}{2} \beta^{2} z\right)^{-1 / 4} \exp \left[\frac{1}{2} \ln \cos (\sqrt{2 / K} t)-\frac{k_{4}^{2}}{4} \int_{t}^{T} \frac{d t}{[\ln \cos (\sqrt{2 / K} t)]^{2}}+k_{5}\right] \\
& \times F(z, t) \exp \left[\frac{\mu}{\beta^{2}}(\alpha \ln z-z) \pm \frac{\sqrt{2} k_{3}}{\ln \cos (\sqrt{2 / K} t)} \sqrt{z}\right],
\end{aligned}
$$

with $k_{j}(j=1, \ldots, 5)$ are integration constants. The terminal condition (5) for the CIR equation is also transformed to

$$
\begin{aligned}
F(z, T)= & \left(\frac{1}{2} \beta^{2} z\right)^{-1 / 4} \exp \left[\frac{1}{2} \ln \cos (\sqrt{K / 2} t)+\frac{\mu^{2} \alpha}{\beta^{2}} T+k_{5}\right] \\
& \times \exp \left\{\frac{\mu}{\beta^{2}}(\alpha \ln z-z) \pm \frac{\sqrt{2} k_{3}}{\ln \cos (\sqrt{K / 2} t)} \sqrt{z}\right\} .
\end{aligned}
$$

In order to seek for the fundamental solutions of the PDE (4), it is seen that there exist equivalence transformations (24) which gives reduction of PDE (4) to the heat equation. This result is employed to find the fundamental solution of the CIR equation.

It is well known that the fundamental solution of the heat is given in barred coordinates [39]:

$$
\bar{F}(\bar{z}, \bar{t})=\frac{1}{2 \sqrt{\bar{t} \pi}} \exp \left[-\frac{\bar{z}^{2}}{4 \bar{t}}\right] .
$$

To look for the fundamental solution of CIR equation (4), solution (26) is transformed by means of (24). With the use of equations (24) and (26), the solution $F(z, t)$ is given by 


$$
\begin{aligned}
F(z, t)= & \left(\frac{1}{2} \beta^{2} z\right)^{1 / 4} \frac{1}{2 \sqrt{t} \pi} \exp \left[-\frac{(z)^{2}}{4 \bar{t}}\right] \\
& \times \exp \left[-\left\{\frac{1}{2} \ln \cos (\sqrt{K / 2} t)-\frac{k_{4}^{2}}{4} \int_{t}^{T} \frac{d t}{[\ln \cos (\sqrt{K / 2} t)]^{2}}+k_{5}\right\}\right] \\
& \times \exp \left[-\left\{\frac{\mu}{\beta^{2}}(\alpha \ln z-z) \pm \frac{\sqrt{2} k_{3}}{\ln \cos (\sqrt{K / 2} t)} \sqrt{z}\right\}\right] .
\end{aligned}
$$

Finally, using the values of $\bar{t}$ and $\bar{z}$ into equation (27), the above solution takes the form

$$
\begin{aligned}
F(z, t)= & \left(\frac{1}{2} \beta^{2} z\right)^{1 / 4} \frac{1}{2 \sqrt{k_{1} \sqrt{K / 2} \tan (\sqrt{K / 2} t) \pi}} \\
& \times \exp \left[-\frac{\left.\left( \pm \sqrt{4 k_{1}} / \cos (\sqrt{K / 2} t) \sqrt{z}+k_{2} \int_{t}^{T} d t / \ln \cos (\sqrt{K / 2} t)+k_{3}\right)^{2}\right]}{4 k_{1} \sqrt{K / 2} \tan (\sqrt{K / 2} t)}\right] \\
& \times \exp \left[-\left\{\frac{1}{2} \ln \cos \left(\sqrt{\frac{K}{2}} t\right)-\frac{k_{4}^{2}}{4} \int_{t}^{T} \frac{d t}{[\ln \cos (\sqrt{K / 2} t)]^{2}}+k_{5}\right\}\right] \\
& \times \exp \left[-\left\{\frac{\mu}{\beta^{2}}(\alpha \ln z-z) \pm \frac{\sqrt{2} k_{3}}{\ln \cos (\sqrt{K / 2} t)} \sqrt{z}\right\}\right] .
\end{aligned}
$$

Under the same set of equivalence transformations for reduction into the heat equation, one can find another closed-form solution for PDE (4). The fundamental solution of the heat equation is also given as series solution of the form [37]

$$
\bar{F}(\bar{z}, \bar{t})=\sum_{r=1}^{\infty} p_{r} \exp \left[-\left(\frac{r \pi}{L}\right)^{2} q \bar{t}\right] \sin \left(\frac{r \pi \bar{z}}{L}\right),
$$

which converges for all $0 \leq x \leq L$. Again, one needs to transform the solution given in equation (29) subject to the transformations given in equation (24). This solution is written as

$$
\begin{aligned}
F(z, t) & =\left(\frac{1}{2} \beta^{2} z\right)^{1 / 4} \sum_{r=1}^{\infty} p_{r} \exp \left[-\left(\frac{r \pi}{L}\right)^{2} q \bar{t}\right] \sin \left(\frac{r \pi \bar{z}}{L}\right) \\
& \times \exp \left[-\left\{\frac{1}{2} \ln \cos \left(\sqrt{\frac{K}{2}} t\right)-\frac{k_{4}^{2}}{4} \int_{t}^{T} \frac{d t}{[\ln \cos (\sqrt{K / 2} t)]^{2}}+k_{5}\right\}\right] \\
& \times \exp \left[-\left\{\frac{\mu}{\beta^{2}}(\alpha \ln z-z) \pm \frac{\sqrt{2} k_{3}}{\ln \cos (\sqrt{K / 2} t)} \sqrt{z}\right\}\right] .
\end{aligned}
$$

Substituting the values of $\bar{z}$ and $\bar{t}$, the series solution $F(z, t)$ takes the form

$$
\begin{aligned}
F(z, t)= & \left(\frac{1}{2} \beta^{2} z\right)^{1 / 4} \sum_{r=1}^{\infty} p_{r} \exp \left[-\left(\frac{r \pi}{L}\right)^{2} q k_{1} \sqrt{\frac{2}{K}} \tan \left(\sqrt{\frac{K}{2}} t\right)\right] \\
& \times \sum_{r=1}^{\infty} \sin \left(\frac{r \pi\left( \pm\left(\sqrt{4 k_{1}} / \cos (\sqrt{K / 2} t)\right) \sqrt{z}+k_{2} \int_{t}^{T} d t / \ln \cos (\sqrt{K / 2} t)+k_{3}\right)}{L}\right) \\
& \times \exp \left[-\left\{\frac{1}{2} \ln \cos \left(\sqrt{\frac{K}{2}} t\right)-\frac{k_{4}^{2}}{4} \int_{t}^{T} \frac{d t}{[\ln \cos (\sqrt{K / 2} t)]^{2}}+k_{5}\right\}\right] \\
& \times \exp \left[-\left\{\frac{\mu}{\beta^{2}}(\alpha \ln z-z) \pm \frac{\sqrt{2} k_{3}}{\ln \cos (\sqrt{K / 2} t)} \sqrt{z}\right\}\right] .
\end{aligned}
$$

2.2. Case 2: Reduction to Second Lie Canonical Equation $-F_{t}=F_{z z}+A / z^{2} F(z, t)$. We look for the case when the CIR PDE (4) is transformed to the second Lie canonical equation. In this situation, one deduce following parametric constraint

$$
\beta^{2} \neq \frac{-8 \mu \alpha \pm 4 \mu \alpha}{-3} \Rightarrow l_{z} \neq 0
$$

Under the same procedure used previously, the equivalence transformations that transform equation (4) into the second Lie canonical form are given by

$$
\begin{aligned}
\bar{t}= & k_{1} \sqrt{\frac{2}{K}} \tan \left(\sqrt{\frac{K}{2}} t\right), \\
\bar{z}= & \pm \frac{\sqrt{8} k_{1}}{\cos (\sqrt{K / 2} t)} \sqrt{z}, \\
\bar{F}= & \sqrt{\cos (\sqrt{K / 2} t)} \exp \left(\alpha t+k_{2}\right) \\
& \exp \left[(\alpha \ln z-z)-\frac{\sqrt{2 K}}{\beta^{2}} \tan (\sqrt{K / 2} t) z\right] F,
\end{aligned}
$$

with $k_{1}$ and $k_{2}$ are the integration constants.

Now, to deduce the closed-form solution such that the CIR equation is transformed into the second Lie canonical 
equation subject to the equivalence transformations given in equation (33), first, rewrite the second canonical equation:

$$
\bar{F}_{\bar{t}}-\bar{F}_{\overline{z z}}-\frac{P}{\bar{z}^{2}} \bar{F}=0 .
$$

In order to find the exact solution of CIR equation, one first needs to seek the solution of PDE (34) and then employ transformations (33). To obtain the solution for PDE (34), the separation of variables technique is used.

Let us assume that the PDE (34) has the separable solution of the form

$$
\bar{F}(\bar{z}, \bar{t})=U(\bar{z}) V(\bar{t}) .
$$

Using equation (35) in equation (34) and separating the variables, one obtains

$$
\begin{gathered}
V_{\bar{t}}+\gamma V=0, \\
U_{\overline{z z}}+\left(\frac{P}{\bar{z}^{2}}+\gamma\right) U=0,
\end{gathered}
$$

with $\gamma \geq 0$ is the separation constant. The following two independent cases are considered here.
Case I. $\gamma=0$

The solutions of ODEs (36) and (37) are written as

$$
\begin{aligned}
& V(\bar{t})=c_{1}, \\
& U(\bar{z})=c_{2} \bar{z}^{1 / 2(1-\sqrt{1-4 P})}+c_{3} \bar{x}^{-1 / 2(1-\sqrt{1-4 P})},
\end{aligned}
$$

Subject to equations (38) and (39), and the exact solution of equation (34) is written as

$$
\bar{F}(\bar{z}, \bar{t})=c_{1}\left[c_{2} \bar{z}^{1 / 2(1-\sqrt{1-4 P})}+c_{3} \bar{x}^{-1 / 2(1-\sqrt{1-4 P})}\right] .
$$

In order to obtain the solution of PDE (4), solution (40) is transformed subject to the transformation relations given in equation (33). One can write the solution $F(z, t)$ as

$$
\begin{aligned}
F(z, t)= & c_{1}\left[c_{2} \bar{z}^{1 / 2(1-\sqrt{1-4 P})}+c_{3} \bar{x}^{-1 / 2(1-\sqrt{1-4 P})}\right], \\
& \times \frac{1}{\sqrt{\cos (\sqrt{K / 2} t)}} \exp \left[\left\{\alpha t+k_{2}\right\}\right] .
\end{aligned}
$$

Substituting the values of $\bar{z}$ and $\bar{t}$ from equation (33) into equation (41), the solution for $F(z, t)$ takes the form

$$
\begin{aligned}
F(z, t)= & c_{1}\left[c_{2}\left( \pm \frac{\sqrt{8} k_{1}}{\cos (\sqrt{K / 2} t)} \sqrt{z}\right)^{1 / 2(1-\sqrt{1-4 P})}+c_{3}\left( \pm \frac{\sqrt{8} k_{1}}{\cos (\sqrt{K / 2} t)} \sqrt{z}\right)^{-1 / 2(1-\sqrt{1-4 P})}\right] \\
& \times \exp \left[-\left\{(\alpha \ln z-z)-\frac{\sqrt{2 K}}{\beta^{2}} \tan \left(\sqrt{\left.\left.\left.\frac{K}{2} t\right) z\right\}\right]}\right.\right.\right. \\
& \times \frac{1}{\sqrt{\cos (\sqrt{K / 2} t)}} \exp \left[\left\{\alpha t+k_{2}\right\}\right] .
\end{aligned}
$$

Case II. $\gamma>0$

Here, the solutions of the ODEs (36) and (37) are found as

$$
\begin{aligned}
V(\bar{t}) & =c_{1} \exp (-\gamma \bar{t}), \\
U(\bar{z}) & =\left[c_{2} \sqrt{\bar{z}} J_{1}\left(\frac{1}{2} \sqrt{1-4 P} ; \sqrt{\gamma} \bar{z}\right)+c_{3} \sqrt{\bar{z}} J_{2}\left(\frac{1}{2} \sqrt{1-4 P} ; \sqrt{\gamma} \bar{z}\right)\right],
\end{aligned}
$$

with $J_{1}$ and $J_{2}$ as the first and the second kind Bessel functions [25], respectively. Therefore, the solution of equation (34) is given by

$$
\bar{F}(\bar{z}, \bar{t})=c_{1} \exp (-\gamma \bar{t})\left[c_{2} \sqrt{\bar{z}} J_{1}\left(\frac{1}{2} \sqrt{1-4 P} ; \sqrt{\gamma} \bar{z}\right)+c_{3} \sqrt{\bar{z}} J_{2}\left(\frac{1}{2} \sqrt{1-4 P} ; \sqrt{\gamma} \bar{z}\right)\right] .
$$

The solution of CIR PDE (4), subject to equation (45), is given as 


$$
\begin{aligned}
F(z, t)= & c_{1} \exp (-\gamma \bar{t})\left[c_{2} \sqrt{\bar{z}} J_{1}\left(\frac{1}{2} \sqrt{1-4 P} ; \sqrt{\gamma} \bar{z}\right)+c_{3} \sqrt{\bar{z}} J_{2}\left(\frac{1}{2} \sqrt{1-4 P} ; \sqrt{\gamma} \bar{z}\right)\right] \\
& \times \frac{1}{\sqrt{\cos (\sqrt{K / 2} t)}} \exp \left[\left\{\alpha t+k_{2}\right\}\right] \\
& \times \exp \left[-\left\{(\alpha \ln z-z)-\frac{\sqrt{2 K}}{\beta^{2}} \tan \left(\sqrt{\frac{K}{2}} t\right) z\right\}\right] .
\end{aligned}
$$

Incorporating the values of $\bar{z}$ and $\bar{t}$ from equation (33) in equation (45), the solution $F(z, t)$ in equation (45) takes the form

$$
\begin{aligned}
F(z, t)= & c_{1} \exp \left(-\gamma k_{1} \sqrt{\frac{2}{K}} \tan \left(\sqrt{\frac{K}{2}} t\right)\right) \\
& \times c_{2} \sqrt{ \pm \frac{\sqrt{8 z} k_{1}}{\cos (\sqrt{K / 2} t)}} J_{1}\left(\frac{1}{2} \sqrt{1-4 P} ; \sqrt{\gamma} \pm \frac{\sqrt{8 z} k_{1}}{\cos (\sqrt{K / 2} t)}\right) \\
& +\left[c_{3} \sqrt{ \pm \frac{\sqrt{8 z} k_{1}}{\cos (\sqrt{K / 2} t)}} J_{2}\left(\frac{1}{2} \sqrt{1-4 P} ; \sqrt{\gamma} \pm \frac{\sqrt{8 z} k_{1}}{\cos (\sqrt{K / 2} t)}\right)\right] \\
& \times \frac{1}{\sqrt{\cos (\sqrt{K / 2} t)}} \exp \left[-\left\{\alpha t+k_{2}\right\}\right] \\
& \times \exp \left[-\left\{(\alpha \ln z-z)-\frac{\sqrt{2 K}}{\beta^{2}} \tan \left(\sqrt{\frac{K}{2}} t\right) z\right\}\right] .
\end{aligned}
$$

\section{Fundamental Solution of Cauchy Initial- Value Problem: A Case Study}

This section is devoted to discuss the fundamental solution of the Cauchy initial-value problem for the CIR model subject to the terminal condition. Since the transformation relations are already deduced for PDE (4) for reduction to heat equation, these transformations are used to discuss the fundamental solutions of the corresponding initial-value problem.

The standard Cauchy initial-value problem for the heat equation is given by [40]

$$
\begin{aligned}
\bar{F}_{\bar{t}} & =\bar{F}_{\overline{z z}}, \\
\bar{F}(\bar{z}, T) & =\Omega(\bar{z}),
\end{aligned}
$$

with some function $\Omega(\bar{z})$. The solution of the above initialvalue problem is given by [38]

$$
\bar{F}(\bar{z}, \bar{t})=\frac{1}{2 \sqrt{\bar{t} \pi}} \int_{-\infty}^{+\infty} \Omega(\eta) \exp \left[-\frac{(\bar{z}-\eta)^{2}}{4 \bar{t}}\right] d \eta .
$$

One needs to transform solution (48) into the solution of CIR equation (4). Inserting $\bar{F}(\bar{z}, \bar{t})$ from equation (48) to equation (33), we deduce

$$
\begin{aligned}
F(z, t)= & \left(\frac{1}{2} \beta^{2} z\right)^{-1 / 4} \exp \left[\left\{\frac{1}{2} \ln \cos \left(\sqrt{\frac{K}{2}} t\right)-\frac{k_{4}^{2}}{4} \int_{t}^{T} \frac{d t}{[\ln \cos (\sqrt{K / 2} t)]^{2}}+k_{5}\right\}\right] \\
& \times \exp \left[\left\{\frac{\mu}{\beta^{2}}(\alpha \ln z-z) \pm \frac{\sqrt{2} k_{3}}{\ln \cos (\sqrt{K / 2} t)} \sqrt{z}\right\}\right] \\
& \times \frac{1}{2 \sqrt{\bar{t} \pi}} \int_{-\infty}^{+\infty} \Omega(\eta) \exp \left[-\frac{(\bar{z}-\eta)^{2}}{4 \bar{t}}\right] d \eta
\end{aligned}
$$

Keeping in mind condition (25), the function $\Omega(\eta)$ in equation (49) is written as

$$
\begin{aligned}
\Omega(\eta)= & \left(\frac{1}{2} \beta^{2} z\right)^{-1 / 4} \exp \left[\frac{1}{2} \ln \cos \left(\sqrt{\frac{K}{2}} t\right)+\frac{\mu^{2} \alpha}{\beta^{2}} T+k_{5}\right] \\
& \times \exp \left\{\frac{\mu}{\beta^{2}}(\alpha \ln z-z) \pm \frac{\sqrt{2} k_{3}}{\ln \cos (\sqrt{K / 2} t)} \sqrt{z}\right\} .
\end{aligned}
$$

Finally, by substituting of the values of $\bar{z}$ and $\bar{t}$ from equation (24) to equation (49), one can write

$$
\begin{aligned}
F(z, t)= & \left(\frac{1}{2} \beta^{2} z\right)^{-1 / 4} \exp \left[-\left\{\frac{1}{2} \ln \cos \left(\sqrt{\frac{K}{2}} t\right)-\frac{k_{4}^{2}}{4} \int_{t}^{T} \frac{d t}{[\ln \cos (\sqrt{K / 2} t)]^{2}}+k_{5}\right\}\right] \\
& \times \exp \left[-\left\{\frac{\mu}{\beta^{2}}(\alpha \ln z-z) \pm \frac{\sqrt{2} k_{3}}{\ln \cos (\sqrt{K / 2} t)} \sqrt{z}\right\}\right] \\
& \times \frac{1}{2 \sqrt{\pi k_{1} \sqrt{K / 2} \tan (\sqrt{K / 2} t)}} \\
& \times \int_{-\infty}^{+\infty} \Omega(\eta) \exp \left[-\frac{\left.\left( \pm\left(\sqrt{4 k_{1}} / \cos (\sqrt{K / 2} t)\right) \sqrt{z}+k_{2} \int_{t}^{T} d t / \ln \cos (\sqrt{K / 2} t)+k_{3}-\eta\right)^{2}\right]}{4 k_{1} \sqrt{K / 2} \tan (\sqrt{K / 2} t)}\right] d \eta
\end{aligned}
$$


The value of the integral in the above solution could be found in terms of the Kummer confluent hypergeometric functions [41] or can be computed numerically.

\section{Conservation Laws of Cox-Ingersoll-Ross Equation}

The conservation laws for the CIR equation (4) are derived in this section. These conservation laws are constructed for the first time by using the conservation theorem of Ibragimov [38]. The CIR equation admits six plus infinite set of Lie symmetries [11]. Every symmetry generator of the CIR equation gives rise to a conservation law.

The formal second-order Lagrangian for the CIR equation (1) is written as

$$
\mathscr{L}=G\left[F_{t}+\frac{1}{2} \beta^{2} z F_{z z}+\mu(\alpha-z) F_{z}-z F\right] .
$$

The Lagrangian defined in (52) is used to derive the adjoint equation of the CIR equation, which is given as

$$
G_{t}+2\left(z+\frac{\beta^{2}}{2}\right) G-\frac{1}{2} z\left[\beta^{2} z G_{\mathrm{zz}}-2 \mu(\alpha-z) G_{z}\right]=0 .
$$
form

Every Lie symmetry generator of the CIR equation of the

$$
\Gamma=\eta_{1} \partial_{t}+\eta_{2} \partial_{z}+\eta_{3} \partial_{F}
$$

gives rise to a conservation law

$$
D_{t}\left(W^{t}\right)+D_{z}\left(W^{z}\right)=0
$$

with

$$
\begin{aligned}
& W^{t}=\eta_{1} \mathscr{L}+C \mathscr{L}_{F_{t}} \\
& W^{z}=\eta_{2} \mathscr{L}+C\left(\mathscr{L}_{\mathrm{Fz}}-D_{z} \mathscr{L}_{F_{\mathrm{zz}}}\right)+D_{z}(C) \mathscr{L}_{F_{\mathrm{zz}}},
\end{aligned}
$$

where

$$
C=\eta_{3}-\left(\eta_{1} F_{t}+\eta_{2}\right) F_{z} .
$$

The symmetries of the CIR equation (4) and the associated adjoint equation can be computed easily. Firstly, we consider the time-translation symmetry $\Gamma_{1}=\partial_{t}$ of CIR equation (4). By using the Ibragimov theorem [38], the components of the conserved vector associated with operator $\Gamma_{1}$ are given by

$$
\begin{aligned}
& W_{1}^{t}=F G_{t}, \\
& W_{1}^{z}=\left(z^{2}-\beta^{2} z\right) F G_{t}+\frac{1}{2} \beta^{2} z^{2}\left(F_{z} G_{t}-F G_{t z}\right) .
\end{aligned}
$$

Similarly, if one uses the symmetry operator $\Gamma_{2}=z \partial_{z}$, one can deduce the associated conservation law with components:

$$
\begin{aligned}
& W_{2}^{t}=-z F_{z} G \\
& W_{2}^{z}=\left[z F_{t}-\mu\left(\alpha z-z^{2}\right) F+\frac{1}{2} \beta^{2} z^{2} F_{z}\right] G+\frac{1}{2} \beta^{2} z^{3} G_{z} .
\end{aligned}
$$

\section{Final Remarks}

The purpose of this work is to demonstrate the utility of an algorithmic method, an invariant approach based on the theory of Lie groups. Utilizing this invariant technique, the valuation parabolic PDE of interest rate derivative in the CIR model framework is studied in detail. The classification of the CIR equation was based on the classical results of Lie's classification theory for the parabolic PDEs. Hence, the invariant theory helped in the computation of equivalence transformations which transform the underlying model to different Lie canonical equations. It was found that, for proper choice of parametric values, the CIR equation was transformed to the first and the second Lie canonical equations. For each case, the transformation relations are derived which reduced the CIR equation into the first and the second Lie canonical form. These transformations were further employed to find the invariant solutions of the CIR equation by using some well-known fundamental solutions of the first and the second Lie canonical equations. Moreover, the Cauchy initial-value problem of the CIR model, with a suitable choice of a terminal condition, is also studied in detail. The solution technique was based on translating the Cauchy initial-value problem for the CIR model into the classical heat equation Cauchy problem and then implementing the well-known Cauchy solutions of the heat equation.

The conservation laws corresponding to the two Lie point symmetries of the CIR equation are constructed by using a general theorem on conservation laws. The notion of conservation laws and associated Lie point symmetries can be used to formulate exact solutions of such type of complicated equations arising in the study of financial mathematics models.

Lie's group theory provides a systematic way to the resolution of the various DEs which arise in financial mathematics. It should come as no surprise that many evolution PDEs in the field of mathematical finance have a rich symmetry group structure. These symmetry structures help to explain the ease with which ansatz-based techniques can yield solutions. However, such nonalgorithmic adhoc procedures have no place, especially when dealing with real-world problems that are described in terms of complicated PDEs. It is important to note that the invariant analysis performed on the CIR equation can be carried out on any other class of parabolic models which transform not only to the heat equation but also to other canonical equations.

\section{Data Availability}

No data were used to support the findings of the study.

\section{Conflicts of Interest}

The author declares that there are no conflicts of interest regarding the publication of this paper. 


\section{References}

[1] L. Bachelier, "Théorie de la spéculation," Annales scientifiques de l'École normale supérieure, vol. 17, pp. 21-86, 1900.

[2] R. C. Merton, "Optimum consumption and portfolio rules in a continuous-time model," Journal of Economic Theory, vol. 3, no. 4 , pp. 373-413, 1971.

[3] F. Black and M. Scholes, "The pricing of options and corporate liabilities," Journal of Political Economy, vol. 81, no. 3, pp. 637-654, 1973.

[4] O. Vasicek, "An equilibrium characterization of the term structure," Journal of Financial Economics, vol. 5, no. 2, pp. 177-188, 1977.

[5] J. C. Cox, J. E. Ingersoll, and S. A. Ross, "An intertemporal general equilibrium model of asset prices," Econometrica, vol. 53, no. 2, pp. 363-384, 1985.

[6] Y. K. Kwok, Mathematical Models of Financial Derivatives, Springer, Singapore, Singapore, 1998.

[7] P. Wilmott, S. Howison, and J. Dewynne, The Mathematics of Financial Derivatives: A Student Introduction, Cambridge University Press, New York, NY, USA, 1995.

[8] W. Sinkala, P. G. L. Leach, and J. G. O'Hara, "Embedding the vasicek model into the cox-ingersoll-ross model," Mathematical Methods in the Applied Sciences, vol. 34, no. 2, pp. 152-159, 2011.

[9] J. Goard, "New solutions to the bond-pricing equation via Lie's classical method," Mathematical and Computer Modelling, vol. 32, no. 3-4, pp. 299-313, 2000.

[10] W. Sinkala, P. G. L. Leach, and J. G. O’Hara, “Zero-coupon bond prices in the vasicek and CIR models: their computation as group-invariant solutions," Mathematical Methods in the Applied Sciences, vol. 31, no. 6, pp. 665-678, 2008.

[11] W. Sinkala, P. G. L. Leach, and J. G. O’Hara, “An optimal system and group-invariant solutions of the Cox-IngersollRoss pricing equation," Applied Mathematics and Computation, vol. 201, no. 1-2, pp. 95-107, 2008.

[12] J.-K. Lee, "An efficient numerical method for pricing American put options under the CEV model," Journal of Computational and Applied Mathematics, vol. 389, Article ID 113311, 2021.

[13] W. Sinkala, "On the derivation of nonclassical symmetries of the black-scholes equation via an equivalence transformation," Mathematical Problems in Engineering.vol. 2020, Article ID 6645568, 7 pages, 2020.

[14] A. H. Davison and S. Mamba, "Symmetry methods for option pricing," Communications in Nonlinear Science and Numerical Simulation, vol. 47, pp. 421-425, 2017.

[15] L. A. Bordag and I. P. Yamshchikov, "Optimization problem for a portfolio with an illiquid asset: Lie group analysis," Journal of Mathematical Analysis and Applications, vol. 453, no. 2, pp. 668-699, 2017.

[16] M. Craddock and M. Grasselli, "Lie symmetry methods for local volatility models," Stochastic Processes and Their Applications, vol. 130, no. 6, pp. 3802-3841, 2020.

[17] N. Habibi, E. Lashkarian, E. Dastranj, and S. R. Hejazi, "Lie symmetry analysis, conservation laws and numerical approximations of time-fractional fokker-planck equations for special stochastic process in foreign exchange markets," Physica A: Statistical Mechanics and Its Applications, vol. 513, pp. 750-766, 2019.

[18] B. C. Kaibe and J. G. O’Hara, "Symmetry analysis of an interest rate derivatives PDE model in financial mathematics," Symmetry, vol. 11, no. 8, p. 1056, 2019.
[19] C. M. Khalique and T. Motsepa, "Lie symmetries, groupinvariant solutions and conservation laws of the vasicek pricing equation of mathematical finance," Physica A: Statistical Mechanics and Its Applications, vol. 505, pp. 871-879, 2018.

[20] O. Patsiuk and S. Kovalenko, "Symmetry reduction and exact solutions of the non-linear black-scholes equation," Communications in Nonlinear Science and Numerical Simulation, vol. 62, pp. 164-173, 2018.

[21] A. Khan, T. Abdeljawad, J. F. Gómez-Aguilar, and H. Khan, "Dynamical study of fractional order mutualism parasitism food web module," Chaos, Solitons \& Fractals, vol. 134, Article ID 109685, 2020.

[22] A. Din, Y. Li, T. Khan, K. Anwar, and G. Zaman, "Stochastic dynamics of hepatitis B epidemics," Results in Physics, vol. 20, Article ID 103730, 2021.

[23] S. Lie, "On integration of a class of linear partial differential equations by means of definite integrals," Archiv for Mathematik og Naturvidenskab, vol. 3, pp. 328-368, 1881.

[24] L. V. Ovsiannikov, Group Properties of Differential Equations, Academic Press, New York, NY, USA, 1982.

[25] G. W. Bluman, "On mapping linear partial differential equations to constant coefficient equations," SIAM Journal on Applied Mathematics, vol. 43, no. 6, pp. 1259-1273, 1983.

[26] I. K. Johnpillai and F. M. Mahomed, "Singular invariant equation for the $(1+1)$ Fokker-Planck equation," Journal of Physics A: Mathematical and General, vol. 34, no. 49, pp. 11033-11051, 2001.

[27] F. M. Mahomed, "Complete invariant characterization of scalar linear $(1+1)$ parabolic equations," Journal of Nonlinear Mathematical Physics, vol. 15, no. 1, pp. 112-123, 2008.

[28] F. Mahomed, K. Mahomed, R. Naz, and E. Momoniat, "Invariant approaches to equations of finance," Mathematical and Computational Applications, vol. 18, no. 3, pp. 244-250, 2013.

[29] A. Bakkaloglu, T. Aziz, A. Fatima, F. M. Mahomed, and C. M. Khalique, "Invariant approach to optimal investmentconsumption problem: the constant elasticity of variance (CEV) model," Mathematical Methods in the Applied Sciences, vol. 40, no. 5, pp. 1382-1395, 2017.

[30] T. Aziz, A. Fatima, and C. M. Khalique, "Integrability analysis of the partial differential equation describing the classical bond-pricing model of mathematical finance," Open Physics, vol. 16, no. 1, pp. 766-779, 2018.

[31] S. C. Anco and G. Bluman, "Derivation of conservation laws from nonlocal symmetries of differential equations," Journal of Mathematical Physics, vol. 37, no. 5, pp. 2361-2375, 1996.

[32] S. C. Anco and G. Bluman, "Direct construction of conservation laws from field equations," Physical Review Letters, vol. 78, no. 15, pp. 2869-2873, 1997.

[33] T. Wolf, "A comparison of four approaches to the calculation of conservation laws," European Journal of Applied Mathematics, vol. 13, no. 2, pp. 129-152, 2002.

[34] S. C. Anco and G. Bluman, "Direct construction method for conservation laws of partial differential equations part I: examples of conservation law classifications," European Journal of Applied Mathematics, vol. 13, no. 5, pp. 545-566, 2002.

[35] A. H. Kara and F. M. Mahomed, "The relationship between symmetries and conservation laws," International Journal of Theoretical Physics, vol. 39, no. 1, pp. 23-40, 2000.

[36] A. H. Kara and F. M. Mahomed, "Noether-type symmetries and conservation laws via partial Lagrangians," Nonlinear Dynamics, vol. 45, no. 3-4, pp. 367-383, 2006. 
[37] A. H. Kara and F. M. Mahomed, "A basis of conservation laws for partial differential equations," Journal of Nonlinear Mathematical Physics, vol. 9, no. 2, pp. 60-72, 2002.

[38] N. H. Ibragimov, "A new conservation theorem," Journal of Mathematical Analysis and Applications, vol. 333, no. 1, pp. 311-328, 2007.

[39] T. Y. N. Myint-U, Partial Differential Equations of Mathematical Physics, Amercian Elsevier Publishing Company, INC., New York, NY, USA, 1973.

[40] J. Hadamard, Lectures on Cauchy's Problem in Linear Partial Differential Equations, Yale University Press, New Haven, CT, USA, 2003.

[41] M. Abramowitz and I. A. Stegun, Handbook of Mathematical Functions, New York, NY, USA, 1965. 